\title{
Respiratory rate estimation from multilead directions, based on ECG delineation
}

\author{
M. Noriega, E.J. Marañón, D. Romero, M. Orini and R. Almeida*†
}

2016

\begin{abstract}
Estimating the instantaneous respiratory rate $(R r)$ from the electrocardiogram (ECG) is of interest as respiration direct measurement in clinical situations is often cumbersome. In this study, the $R r$ was estimated from the same Final Directions of maximum projection (FD) used for multi lead ECG automatic delineation. Power spectral analysis over the directions based on QRS complex main peak and T wave on-set, peak and end spatial loops was used for $R r$ estimation. On a subset of the Physionet MGH/MF dataset, the proposed method yielded more accurate $R r$ estimates (minimum mean absolute error (MAE), $2.82 \mathrm{bpm}$ ) than the frequency tracking algorithm (minimum MAE, $4.53 \mathrm{bpm}$ ) and Fourier-based frequency estimation (minimum MAE, $4.94 \mathrm{bpm}$ ) using each lead alone, outperforming also the weighted multi-signal oscillator-based algorithm estimates for two or three lead (minimum MAE, $3.04 \mathrm{bpm}$ ). It was also shown that the FD of the three orthogonalized leads from Principal Component algorithm, improve the performance of $R r$ estimation.
\end{abstract}

\section{Introduction}

The respiratory rate $(R r)$ needs to be monitored in different applications, which can be done estimating it directly over the respiratory signal itself, or indirectly from other biological signals such as the ECG. The interest of the indirect estimation of $\mathrm{Rr}$ appear because the direct estimation is done with devices that are intrusive, expensive and uncomfortable for the patient [1-3]. It is well known that respiratory and cardiac activities are related by physiological processes. The respiration modulates the heart rate such that it increases during inspiration and decreases during expiration [4]. In the same way, during the respiratory cycle, chest movements and changes in the thorax impedance distribution due to filling and emptying of the lungs cause a rotation of the electrical axis of the heart which has an effect over beat morphology $[5,6]$. Several studies have developed signal processing techniques to extract respiratory information from the modulation of the ECG morphology and heart rate, using both time and frequency based methods [3, 5,7-9]. However, the R-peak amplitudes (RPA) based Rr estimates depend on the given ECG lead, as beat respiratory related morphological changes differ lead to lead and most influenced lead is subject dependent. When several ECG leads are available methods relying in multilead QRS area or vectocardiographic (VCG) loops are preferable [5].

Often in ambulatory and clinical applications multilead ECG recordings are available. This make possible to construct ECG loops based in orthogonal or orthogonalized no parallel leads. The main directions on the wavelet transform (WT) of those loops, token as parallel to each fiducial point, have been successfully used for multilead delineation [10]. Moreover a strong relation between the respiration and the $\mathrm{T}$ wave end based Final Directions of maximum projection (FD) used the by multilead ECG delineation system (ML) was found in [11]. In the present study, we propose and validate a method for $R r$ estimation from the FD found in the delineation of QRS peak and T wave on-set, peak and end.

${ }^{*}$ M. Noriega is with the Comunications and Electronical Departament of Oriente University, Santiago de Cuba (Cuba), (e-mail: maikeln@uo.edu.cu)E.J. Marañón is with the Studies's Center of Neuro-science and Signal and Image Processing (CENPIS),(e-mail: enriquem@uo.edu.cu). D. Romero is with the Laboratoire Traitement du Signal et de l'Image, Université de Rennes 1, Rennes, France, (e-mail: daniel.romero@univ-rennes1.fr). Orini, Michele is with the University College London (e-mail: m.orini@ucl.ac.uk). R. Almeida (correspondent author) is with CMUP - Centro de Matemática da Universidade do Porto, FCUP, Porto, Portugal; BSICoS Group, Aragón Institute of Engineering Research (I3A), University of Zaragoza, Zaragoza, Aragón, Spain and The Biomedical Research Networking center in Bioengineering, Biomaterials and Nanomedicine, Spain (email: rbalmeid@fc.up.pt).

${ }^{\dagger}$ This study was partially supported by projects TEC2010-21703-C03-02 and TEC2013-42140-R from the Ministerio de Economia y Competitividad (MINECO) with European Regional Development Fund (FEDER), Spain, and by Grupo Consolidado BSICoS (T-96) from DGA (Aragón, Spain) and European Social Fund (EU). D. Romero acknowledges the financial support of the Fondation LefoulonDelalande, Institut de France, France. M. Orini was supported by a Marie Curie IEF-2013 fellowship. R. Almeida was supported by CMUP (UID/MAT/00144/2013), which is funded by FCT (Portugal) with national (MEC) and European structural funds through the programs FEDER, under the partnership agreement PT2020. 


\section{Materials and Methods}

\section{$2.1 \quad$ Data}

For the sake of comparison, the evaluation data used in this study was the same as in [12]: a subset of 20 different lengths recordings ( 7 female, 13 male, aged 29-84) from the Physionet MGH/MF waveform database [13,14] in total of 41.73 hours, with characteristics reported Table I. This dataset was recorded from stable and unstable patients at the Massachusetts General Hospital at various physiological conditions. The selected subset of interest to this study contains the respiratory impedance, ECG leads I, II and an unidentified V lead, digitized at a rate of $360 \mathrm{~Hz}$. Reference $R r$ values reported by [12] are included in Table 1.

Table 1: Evaluation data charateristics as in [12]. Cardiac Rhythm - SR: sinus rhythm, ST: sinus tachycardia, SB: sinus bradycardia, VP: ventricular pacing, AP: atrial pacing, AF: atrial fibrillation, AFL: atrial flutter, JR: junctional rhythm. Respiratory condition - S: spontaneous, C: controlled, IMV: intermittent mandatory ventilation. For IMV, the $R r$ range is reported.

\begin{tabular}{cccc}
\hline \hline & $\begin{array}{c}\text { Cardiac } \\
\text { Rhythm }\end{array}$ & $\begin{array}{c}\text { Respiratory } \\
\text { Condition }\end{array}$ & $R r(\mathrm{bpm})$ \\
\hline mgh005 & ST & C & 12 \\
\hline mgh006 & VP & IMV & $8-22$ \\
\hline mgh007 & SR & S & 16 \\
\hline mgh008 & AF & S & 16 \\
\hline mgh009 & ST & IMV & $6-20$ \\
\hline mgh013 & AF & S & 20 \\
\hline mgh014 & AP & S & 18 \\
\hline mgh016 & VP & IMV & $2-18$ \\
\hline mgh020 & JR & C & 7 \\
\hline mgh024 & AFL & S & 16 \\
\hline mgh026 & ST & S & 16 \\
\hline mgh027 & AF & S 18 & \\
\hline mgh028 & VP & S & 20 \\
\hline mgh029 & ST & C & 10 \\
\hline mgh030 & AF & C & 18 \\
\hline mgh031 & ST & S & 30 \\
\hline mgh034 & SB & S & 16 \\
\hline mgh035 & SB & IMV & $5-8$ \\
\hline mgh037 & SR & S & 16 \\
\hline mgh038 & SR & S & 16 \\
\hline \hline
\end{tabular}

\subsection{Estimated breathing pattern from ML ECG delineation}

ML delineation system considered was previously proposed and validated by Almeida R. in [10] and is included in the user-friendly interface in Matlab®, BioSigBrowser [15]. It uses simultaneously two or three orthogonal leads of the ECG (VCG loops) to define spacial WT loops. Denoting the WT of a signal $s(n) \in\{x(n), y(n), z(n)\}$ at scale $\mathrm{m}$ by $w_{s, m}[n]$, the spatial WT loop is defined as:

$$
\mathbf{w}_{m}(n)=\left[w_{x, m}(n), w_{y, m}(n), w_{z, m}(n)\right]^{T}
$$

As a consequence of the WT prototype used, the WT loop $\left.\mathbf{w}_{m}(n)\right|_{n \in L}$ is proportional to the VCG derivative and describes the velocity of evolution of the electric heart vector $(\mathrm{EHV})$ in a time interval $L$. Assuming that the noise is spatially homogeneous, the direction with maximum projection of the WT in the region close to the fiducial point of interest would define the ECG lead maximizing the local SNR, and thus, the most appropriate for the delineation. The main direction $\mathbf{u}=\left[\mathrm{u}_{x}, \mathrm{u}_{y}, \mathrm{u}_{z}\right]^{T}$ of EHV variations on any time interval $L$ is given by the director vector of the best straight linear fit to all points in the WT loop. By choosing adequately the time interval $L$, around the fiducial point of interest, it is possible to find the direction $\mathbf{u}$ corresponding to the lead most suited for delineation purposes [10], here called Final Directions of maximum projection (FD).

The projection of the WT loop $\left(\mathbf{w}_{m}(n)\right)$ over the direction $\mathbf{u}$ allows to obtain a derived wavelet signal $w_{d, m}(n)$ that combines the information provided by the 3 or 2 leads:

$$
w_{d, m}(n)=\frac{\mathbf{w}_{m}^{T}(n) \cdot \mathbf{u}}{\|\mathbf{u}\|} ; n \in I
$$


The time intervals $I$ (used for projecting) and $L$ (used for linear fitting) can be different, depending on each wave specificities.

The strategy proposed for ML boundary delineation using WT loops is based in a multi-step iterative search for a better spatial lead (with steeper slopes) for each boundary delineation. The goal is to construct a derived wavelet signal well suited for boundaries location [16], using the same detection criteria as in the SL delineator proposed in [17].

As we have shown in [11], the direction $\mathbf{u}$ based in the $\mathrm{T}$ wave end relates closely with the respiratory signal. In this study we aim to obtain the signal corresponding to the breathing pattern from the same direction $\mathbf{u}$ used for delineation, taking advantage of this relation. The directions found in the delineation of QRS peak and $\mathrm{T}$ wave on-set, peak and end ( $\mathbf{u}_{h}$ where $h \in R_{p}, T_{o}, T_{p}, T_{e}$, respectively) were considered comparatively.

As the leads recorded in Physionet MGH/MF database, are not orthogonal, they must be orthogonalized before applying the ML delineation system. In this paper three variants orthogonalization is used:

Construction of a pair of orthogonal leads from two known leads (O2KL): As matter of fact, any hypothetical lead in a plane can be synthesized from a lead system with at least two no parallel leads in that plane. In this case, a new ECG lead orthogonal, not necessarily from the standard system, is constructed from the provided leads. Notice that with this approach only 2 dimensional loops are considered.

Orthogonalization of the three ECG lead by Gram-Schmidt algorithm (O3GS): In this case the orthogonalization is performed using the Gram-Schmidt algorithm [18]. We consider all the three leads as vectorial spaces. The synthesized leads are not necessarily in the frontal or transverse plane used to define the standard lead system. Obtaining three orthogonal leads using principal components decomposition (O3PC): In this alternative is considered the VCG defined by the three principal components (PC) obtained from the 3 available leads. The PC are ortogonal ECG like signals which can be interpreted as synthesized leads that are not necessarily in the frontal or transverse plane used to define the standard lead system.

The application of the ML delineation strategy to the 20 records was then performed over the orthogonalized leads using each of the three variants. From this delineation are obtained the vectors defining FD for each heartbeat corresponding to the directions of interestnof the fiducial points considered $\left(\mathbf{u}_{h}(i)\right.$, where $\left.h \in R_{p}, T_{o}, T_{p}, T_{e}\right)$. The coordinates of each $\mathbf{u}$, originally with one value per heart beat, were re-sampled uniformly at $2 \mathrm{~Hz}$ using cubic spline interpolation.

\subsection{Estimating the $R_{r}$ from directions $\mathrm{u}$}

The $R_{r}$ estimation is performed spectrally from interpolated directions $\mathbf{u}_{h}$. Estimation of the power spectrum is accomplished with Burg's method [19]. The spectrum of each directions series $\left(U_{h}(f)\right)$ is estimated with nonoverlapping windows of $60 \mathrm{~s}$ duration. Individual running power spectra of each direction are averaged in order to reduce the frequential peaks not related to breathing. The respiratory frequency for each fiducial point studied was estimated on average spectrum for each window $\mathrm{n}\left(f_{h}(n)\right)$. Estimation of the respiratory frequency as the largest peak of $U_{h}(f)$ comes with the risk of choosing the location of a spurious peak. This risk is, however, considerably reduced by narrowing down the search interval to only include frequencies between $0.15 \mathrm{~Hz}$ and $0.45 \mathrm{~Hz}$ of respiratory frequency. The $R r$ in breaths-per-minute (bpm) is calculated from the respiratory frequencies:

$$
R r_{h}(n)=60 * f_{h}(n)
$$

\subsection{Estimation of the reference $R_{r}$}

To evaluate the $R r$ estimated from the directions $\mathbf{u}$ one needs to compare it with the $R r$ estimated with Burg's method as from the respiratory impedance waveform $\left(R r_{r i w}\right)$. Prior to the reference $R r$ estimation process, the respiratory impedance waveform was re-sampled uniformly at $2 \mathrm{~Hz}$ using cubic spline interpolation and band-pass filtered between $0.1 \mathrm{~Hz}$ and $0.5 \mathrm{~Hz}$.

In addition, as in [12], in this study five frequency estimation algorithms were used to estimate an additional reference $R r\left(R r_{t r u}\right)$ from the Physionet MGH/MF respiratory signals. The five methods used in this study are Fourier maximum frequency estimate, the number of respiratory peaks in 60 second-long centered windows, Frequency estimate using the empirical mode decomposition followed by the Hilbert transform (EMD) [20], the inverse of the time-lapse between two consecutive respiratory peaks and frequency estimate based on the autocorrelation [21]. The median of the five estimates and the two estimates closest to it were averaged and low pass filtered to produce the final reference $R r_{t r u}$.

The accuracy of the ECG-based $R r$ estimates were evaluated by computing their mean absolute error (MAE) in terms of breaths-per-minute with respect to the $R r_{r e f}$, where $r e f \in$ riw, tru. Window-based errors were computed, 


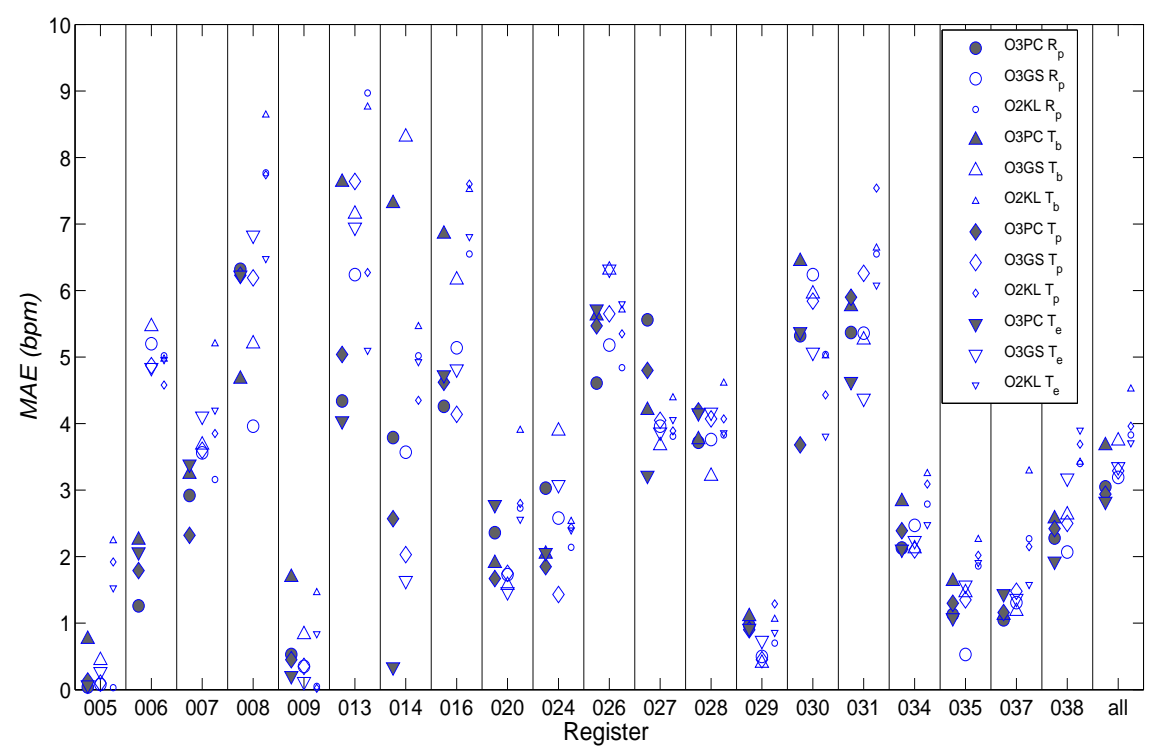

Figure 1: The MAE values in bmp with respect to $R r_{r i w}$, from the directions u estimated with each of the three ortogonalization methods.

where both the estimated $R r$ and the $R r_{r e f}$ were averaged in $60 \mathrm{~s}$ windows.

$$
M A E=\frac{1}{M} \sum_{n=1}^{M}\left|R r_{h}(n)-R r_{r e f}(n)\right|
$$

where $M$ is the number of windows in which the window-based error is computed, $R r_{h}(n)$ is the estimated $R r$ and $R r_{r e f}(n)$ is the reference $R r$.

\section{Results and discussion}

Figure 1 shows the MAE values (per register and considering all files) in bmp with respect to $R r_{\text {riw }}$, from directions $\mathbf{u}$ estimated with each of the three ortogonalization methods. Table 2 reports the MAE: across files, estimated with the three orthogonalization methods and the two reference estimate methods. The MAE of the $R r$ estimated using O3PC orthogonalization were found to be typically lower than both the errors of the O3GS and O2KL. Notice that for O2KL by having only two leads, some information is lost and the error is expected to be greater. This loss have

Table 2: Errors average (bpm) across files of estimated $R_{r}$ for each orthogonalization method (OM) - 2 orthogonal leads from 2 known leads (O2KL), Gram-Schmidt algorithm (O3GS), principal components (O3PC) -, with respect to the reference values based on respiratory impedance waveform: $R r_{r i w}$ (Burg estimation) or $R r_{t r u}$ (combination of 5 estimations).

\begin{tabular}{cccccc}
\hline \hline OM & $\begin{array}{c}\text { reference } \\
\text { estimate method }\end{array}$ & $R r_{R_{p}}$ & $R r_{T_{b}}$ & $R r_{T_{p}}$ & $R r_{T_{e}}$ \\
\hline O2KL & $R r_{\text {riw }}$ & 3.83 & 4.52 & 3.96 & 3.71 \\
O2KL & $R r_{\text {tru }}$ & 3.80 & 4.50 & 3.90 & 3.67 \\
O3GS & $R r_{\text {riw }}$ & 3.19 & 3.74 & 3.29 & 3.35 \\
O3GS & $R r_{\text {tru }}$ & 3.16 & 3.71 & 3.27 & 3.34 \\
O3PC & $R r_{\text {riw }}$ & 3.05 & 3.67 & 2.94 & $\mathbf{2 . 8 3}$ \\
O3PC & $R r_{\text {tru }}$ & 3.02 & 3.64 & 2.93 & $\mathbf{2 . 8 2}$ \\
\hline \hline
\end{tabular}

been already reported in $\mathrm{T}$ wave end delineation in [22]. In respect to the $\mathrm{Rr}$ estimated based in different fiducial points, best results are obtained for Te, in two out of the three orthogonalization methods (O3PC and O2KL. This result is surprising as QRS complex, as more preeminent, is usually used as reference for morphologic changes and electrical axes rotation. It could be the case that the ML lead system for delineation is more well tuned for $\mathrm{T}$ wave end FD search or that the FD is more unstable for main QRS wave loop. In spite of the best combination orthogonalization method / fiducial point varied across files, O3PC using the direction defined for $T_{e}$ delineation can 
be considered as presenting the best global performance for obtaining the $R r$.

For the sake of comparison, the Table 3 contains the minimum global MAE for the Fourier based estimates (FB), oscillator-based adaptive frequency tracking algorithm (OSC) estimates for each lead and the weighted multi-signal oscillator-based algorithm (W-OSC) estimates for two or three leads reported by [12].

Table 3: Minimum average error in bpm obtained in [12] from the RPA signals of the Physionet MGH/MF database.

\begin{tabular}{cc}
\hline \hline $\begin{array}{c}R r \text { estimation } \\
\text { method }\end{array}$ & $\begin{array}{c}\text { minimum } \\
\text { average } M A E\end{array}$ \\
\hline FB & 4.94 \\
OSC & 4.53 \\
W-OSC & 3.04 \\
\hline
\end{tabular}

Note that for both single lead based methods, FB and OSC, all average MAE obtained are higher than any global MAE obtained from the directions $\mathbf{u}$ of ML. For the multilead based method W-OSC, best result is equivalent to the value obtained with O3PC for the main QRS wave, but higher than the values for T wave peak and end.

\section{Conclusion}

In this study, we have shown that it is possible to extract accurately the $R r$ from directions $\mathbf{u}$, obtained from the ML delineator. Thus, the beat-to-beat estimation of respiratory rate can be obtained as an extra output of ML delineation with almost no extra effort, allowing easy, unintrusive, cheap and possibly ambulatory respiratory monitoring, with no extra discomfort for the patient. Additionally is evidenced that the best estimate of the $R r$ is obtained when using the orthogonalization with principal component decomposition. It was also verified that using only two orthogonal leads spatial resolution is lost and therefore the accuracy decreased. Finally, the mean absolute errors for the strategy using the $\mathrm{T}$ wave end based final direction obtained in this work, are lower that the best results reported with the OSC and FB algorithms applied to each lead and than the weighted multi-signal oscillator-based algorithm (W-OSC, outperforming R-peak amplitudes based approaches the reported in the literature.

\section{References}

[1] M.A. Cretikos, R. Bellomo, K. Hillman, J. Chen, S. Finfer, A. Flabouris, "Respiratory rate: the neglected vital sign", Med. J. Aust. 188 (11) (2008) 657.

[2] L. Brochard, G.S. Martin, L. Blanch, P. Pelosi, F.J. Belda, A. Jubran, L. Gattinoni, J. Mancebo, V.M. Ranieri, J.-C.M. Richard, et al., "Clinical review: respiratory monitoring in the ICU" -a consensus of 16, Crit. Care 16 (2) (2012) 219.

[3] Mirmohamadsadeghi L, Vesin J.M. "Respiratory rate estimation from the ECG using an instantaneous frequencytracking algorithm". Biomed Signal Process Control 2014; 14:66-72.

[4] Yasuma F, Hayano J. "Respiratory sinus arrhythmia: Why does the heartbeat synchronize with respiratory rhythm?" Chest. 2004; 125(2):683-90.

[5] R. Bailón, L. Sörnmo, P. Laguna. "ECG-derived respiratory frequency estimation". In F. Azuaje GD. Clifford and PE. McSharry, editors, Advanced methods and tools for ECG data analysis. (2006), pp. 215 - 244. Artech House, Inc.

[6] Aström, M., H. C. Santos, L. Sörnmo, P. Laguna and B. Wohlfart, 2000. "Vectorcardiographic loop alignment and the measurement of morphologic beat-to-beat variability in noisy signals." IEEE Trans. Biomed. Eng. 47(4), 497-506.

[7] Cysarz, Zerm R, Bettermann H, Frtihwirth M, Moser M, Kroz M. "Comparison of respiratory rates derived from heart rate variability, ECG amplitude, and nasal/oral airflow". Ann Biomed Eng. 2008; 36:2085-2094.

[8] Orphanidou C, Fleming S, Shah S, Tarassenko L. "Data fusion for estimatig respiratory rate from a single-lead ECG". Biomed Signal Process Control 2013; 8:98-105. 
[9] Prudat Y, Vesin JM. "Multi-signal extension of adaptive frequency tracking algorithms". Signal Process. 2009; 89:963-973.

[10] Almeida R, Martínez JP, Rocha AP, Laguna P. "Multilead ECG delineation using spatially projected leads from wavelet transform loops." IEEE Trans. Biomed. Eng., vol 56, no 8, 2009, pp 1996-2005.

[11] Noriega, M.; Martínez, J. P. ; Laguna, P. ; Bailón, R. ; Almeida, R.: "Respiration Effect on Wavelet-Based ECG T-Wave End Delineation Strategies", IEEE Trans. Biomed. Eng., vol 59, 2012, pp 1818-1828.

[12] Leila Mirmohamadsadeghi and lean-Marc Vesin, "Respiratory Rate Estimation from Multi-Lead ECGs using an Adaptive Frequency Tracking Algorithm," in: Computers in Cardiology. IEEE Computer Society Press, 2014; 41:353-356.

[13] Welch J, Ford P, Teplick R, Rubsamen R. "The Massachusetts General Hospital-Marquette Foundation hemodynamic and electrocardiographic databasecomprehensive collection of critical care waveforms". J Clin Monit 1991; 7:96-97.

[14] Goldberger A, Amaral L, Glass L, Hausdorff J, Ivanov P, Mark R, et al. "PhysioBank, PhysioToolkit, and PhysioNet: Components of a new research resource for complex physiologic signals". Circulation. 2000; 101:215220 .

[15] J. Bolea and R. Almeida and P. Laguna and L. Sörnmo and J. P. Martínez,: "BioSigBrowser, biosignal processing interface". 9th International Conference on Information Technology and Applications in Biomedicine, ITAB 2009; pp 1-4.

[16] S. Mallat and S. Zhong,: "Characterization of signals from multiscale edge," IEEE Trans. Pattern Anal. Machine Intell., vol. 14, July 1992, pp. 710-732.

[17] Martínez JP, Almeida R, Olmos S, Rocha AP, Laguna P. "Wavelet-based ECG delineator: evaluation on standard databases." IEEE Trans. Biomed. Eng., vol 51, 2004, pp 570-81.

[18] G.H. Golub, "Numerical methods for solving linear least squares problems", Numer. Math. (1965); 7:206-216 .

[19] Marple, S.L. "Digital Spectral Analysis", Englewood Cliffs, NJ, Prentice-Hall, 1987, Chapter7.

[20] E. Huang, Z. Shen, S.R. Long, M.C. Wu, H.H. Shih, Q. Zheng, N.-C. Yen, C.C. Tung, H.H. Liu, "The empirical mode decomposition and the Hilbert spectrum for non-linear and non-stationary time series analysis", Proc. R. Soc. London A: Math. Phys. Eng. Sci. 454 (1971) (1998) 903-995.

[21] Yan Cao, Gang Wei, Fang-Jiong Chen. "A closed-form expanded autocorrelation method for frequency estimation of a sinusoid". Signal Process. 2012; 92:885-892.

[22] Almeida, R.; Martinez, J.P.; Rocha, A.P.; Laguna, P., "Are 2 electrocardiographic leads enough for multilead wave boundary location and QT measuring?," in Computers in Cardiology, 2009 , vol., no., pp.593-596, 13-16 Sept. 2009 